\title{
On the Lindley Record Values and Associated Inference
}

\author{
A. Fallah ${ }^{1}$, A. Asgharzadeh ${ }^{2, *}$, and S.M.T.K. MirMostafaee ${ }^{2}$ \\ ${ }^{1}$ Department of Statistics, Faculty of Mathematics and Statistics, Payame Noor University, Tehran, Iran \\ ${ }^{2}$ Department of Statistics, Faculty of Mathematical Sciences, University of Mazandaran, Babolsar, Iran
}

\section{ARTICLE INFO}

Article History

Received 15 Jul 2016

Accepted 2 Nov 2017

Keywords

Best linear invariant estimators Best linear unbiased estimators

Lindley distribution

Double moments

Pivotal quantity

Prediction

Record values

Single moments.

2010 Mathematics Subject Classification

62F15, 62F25, 68U20

\section{ABSTRACT}

In this paper, we discuss the record values arising from the Lindley distribution. We compute the means, variances and covariances of the record values. These values are used to compute the best linear unbiased estimators (BLUEs) and the best linear invariant estimators (BLIEs) of the location and scale parameters. By using the BLUEs and BLIEs, we construct confidence intervals for the location and scale parameters through Monte Carlo simulations. Prediction for the future records is also discussed.

\section{INTRODUCTION}

Let $X_{1}, X_{2}, \cdots$ be a sequence of independent and identically distributed (IID) random variables with cumulative distribution function (cdf) $F(x)$ and probability density function (pdf) $f(x)$. An observation $X_{j}$ is an upper record value of this sequence if it exceeds in value all preceding observations, i.e., if $X_{j}>X_{i}, \forall i<j$. Lower records are analogously defined. Generally, if $\{U(n), n \geq 1\}$ is defined by

$$
U(1)=1, \quad U(n)=\max \left\{j: j<U(n-1), X_{j}>X_{U(n-1)}\right\},
$$

then the sequence $\left\{X_{U(n)}, n \geq 1\right\}$ provides a sequence of upper record statistics. The sequence $\{U(n), n \geq 1\}$ represents the upper record times. From the above definition, the sequence of record statistics can be viewed as order statistics from a sample whose size is determined by the values and the order of occurrence of the observations. Note that from a sequence of $n$ IID continuous random variables, only about $\log (n)$ records are expected, see Houchens [1].

Chandler [2] defined the model of record statistics as a model for successive extremes in a sequence of IID random variables. These statistics are of interest and important in many real life applications involving data relating to weather, economics, sport and life testing studies. For more details and applications regarding record values, see Ahsanullah [3], Arnold et al. [4] and Nevzorov [5].

Recently, the Lindley distribution has received a considerable attention in the statistical literature. It was first proposed by Lindley [6] in the context of Bayesian inference. The pdf of the Lindley distribution is given by

$$
f(x)=\frac{\theta^{2}}{\theta+1}(1+x) e^{-\theta x}, \quad x>0, \quad \theta>0,
$$

*Corresponding author. Email: a.asgharzadeh@umz.ac.ir 
and the corresponding cdf is

$$
F(x)=1-\frac{\theta+1+\theta x}{\theta+1} e^{-\theta x}, \quad x>0, \quad \theta>0 .
$$

A distribution that is close in form to Eq. (2) is the well-known exponential distribution with pdf

$$
f(x)=\theta e^{-\theta x}, \quad x>0, \quad \theta>0 .
$$

Ghitany et al. [7] showed in many ways that the Lindley distribution is a better model than one based on the exponential distribution. The shape of the hazard rate function of the Lindley distribution is an increasing function. This distribution belongs to an exponential family and it can be written as a mixture of an exponential and a gamma distribution with shape parameter 2 . In recent years, this distribution has been studied and extended by many authors $[7 ; 8 ; 9 ; 10 ; 11 ; 12 ; 13 ; 14]$.

Recently, some work has been done on inferential procedures for the Lindley distribution based on complete and censored data [15; 16; 17; 18; 19]. Recently, Asgharzadeh et al. [20] discussed the maximum likelihood and Bayesian estimation of the shape parameter of the Lindley distribution based on upper records. In this article, we consider the upper record values from the Lindley distribution. We compute the means, variances and covariances of the upper record values. Then, we use these moments to calculate best linear unbiased estimators (BLUEs) and best linear invariant estimators (BLIEs) for the location and scale parameters of the Lindley distribution. Prediction for the future records is also discussed. Ahsanullah [21] and Dunsmore [22] discussed the BLUEs and prediction of future record values from a two-parameter exponential distribution. Some work in this direction has been done for the logistic distribution by Balakrishnan et al. [23], for the normal distribution by Balakrishnan and Chan [24], for the generalized exponential distribution by Raqab [25], for the gamma distribution by Sultan et al. [26] and for the Nadarajah-Haghighi distribution by MirMostafaee et al. [27].

In this paper, we consider the upper record values from the Lindley distribution. In Section 2, we compute the means, variances and covariances of record values up to sample size 6. Next, in Section 3, we obtain the BLUEs and BLIEs of the location and scale parameters of the Lindley distribution. The BLUEs and BLIEs are then used to construct the confidence intervals (CIs) for the location and scale parameters. In Section 4, we discuss point and interval predictions for future records. In Section 5, two numerical examples are given to illustrate the estimation and prediction methods discussed in this paper. In Section 6, Monte Carlo simulations are performed to compare the proposed CIs of the location and scale parameters and also to compare the prediction intervals (PIs) of the future records.

\section{MOMENTS OF THE UPPER RECORD VALUES}

Let $X_{U(1)}, X_{U(2)} \cdots, X_{U(n)}$ be the first $n$ upper record values from the Lindley distribution. Then the pdf of $X_{U(n)}$ is given by [4]

$$
f_{n}(x)=\frac{1}{\Gamma(n)}[-\log \{1-F(x)\}]^{n-1} f(x), \quad x>0, \quad n=1,2, \cdots
$$

where $f(\cdot)$ and $F(\cdot)$ are given by Eqs. (2) and (3), respectively. The joint pdf of $X_{U(m)}$ and $X_{U(n)}$ is given by

$$
\begin{aligned}
f_{m, n}(x, y)= & \frac{1}{\Gamma(m) \Gamma(n-m)}[-\log \{1-F(x)\}]^{m-1} \frac{f(x)}{1-F(x)} \times[-\log \{1-F(y)\}+\log \{1-F(x)\}]^{n-m-1} f(y) \\
& 0<x<y<\infty, \quad m=1,2, \cdots, m<n
\end{aligned}
$$

Then, the $r$ th single moment of $X_{U(n)}$ denoted by $\mu_{n}^{(r)}(r=0,1,2, \ldots)$ is given by

$$
\begin{aligned}
\mu_{n}^{(r)}=E\left(X_{U(n)}^{r}\right) & =\int_{0}^{\infty} x^{r} f_{n}(x) \mathrm{d} x \\
& =\int_{0}^{\infty} x^{r} \frac{\theta^{2}(1+x)}{\Gamma(n)(\theta+1)}\left[-\log \left(\frac{\theta+1+\theta x}{\theta+1}\right)+\theta x\right]^{n-1} e^{-\theta x} \mathrm{~d} x \\
& =\frac{1}{(n-1) !} \sum_{i=0}^{n-1}\left(\begin{array}{l}
n-1 \\
i
\end{array}\right) g(i, n, r, \theta),
\end{aligned}
$$


where

$$
g(i, n, r, \theta)=\frac{\theta^{2}}{\theta+1} \int_{0}^{\infty} x^{r}[-\log \{1+\theta x /(\theta+1)\}]^{i}(\theta x)^{n-i-1}(1+x) e^{-\theta x} \mathrm{~d} x
$$

Note that using the transformation $y=1+\theta x /(\theta+1)$, we can write $g(i, n, r, \theta)$ as

$$
\begin{aligned}
g(i, n, r, \theta)= & (\theta+1)^{n-i+r-1} \theta^{-r} e^{\theta+1} \int_{1}^{\infty}[-\log y]^{i}(y-1)^{n-i+r-1} e^{-(\theta+1) y}[(\theta+1) y-1] \mathrm{d} y \\
= & (\theta+1)^{n-i+r} \theta^{-r} e^{\theta+1} \\
& \times\left[\sum_{q=1}^{n-i+r+1}\left(\begin{array}{l}
n-i+r \\
q-1
\end{array}\right)(-1)^{n+r-q+1} \int_{1}^{\infty}[\log y]^{i} y^{q-1} e^{-(\theta+1) y} \mathrm{~d} y\right. \\
& \left.+\frac{\theta}{\theta+1} \sum_{q=1}^{n-i+r}\left(\begin{array}{l}
n-i+r-1 \\
q-1
\end{array}\right)(-1)^{n+r-q} \int_{1}^{\infty}[\log y]^{i} y^{q-1} e^{-(\theta+1) y} \mathrm{~d} y\right] \\
= & (\theta+1)^{n-i+r} \theta^{-r} e^{\theta+1} \\
& \times\left[\sum_{q=1}^{n-i+r+1}\left(\begin{array}{l}
n-i+r \\
q-1
\end{array}\right)(-1)^{n+r-q+1} \frac{\partial^{i}}{\partial q^{i}}\left\{(\theta+1)^{-q} \Gamma(q, \theta+1)\right\}\right. \\
& \left.+\frac{\theta}{\theta+1} \sum_{q=1}^{n-i+r}\left(\begin{array}{l}
n-i+r-1 \\
q-1
\end{array}\right)(-1)^{n+r-q} \frac{\partial^{i}}{\partial q^{i}}\left\{(\theta+1)^{-q} \Gamma(q, \theta+1)\right\}\right],
\end{aligned}
$$

where the last equality is calculated by considering the Eq. (4.358.1) in Gradshteyn and Ryzhik [28]. Here $\Gamma(\cdot, \cdot)$ denotes the incomplete gamma function given by

$$
\Gamma(\nu, \lambda)=\int_{\lambda}^{\infty} x^{v-1} e^{-x} \mathrm{~d} x
$$

Let us now consider the double moments of the upper record values $X_{U(m)}$ and $X_{U(n)}, m<n$. The double $(r, s)$ th moment of $X_{U(n)}$ and $X_{U(n)}$ is given by

$$
\begin{gathered}
\mu_{m, n}^{(r, s)}=E\left(X_{U(m)}^{r} X_{U(n)}^{s}\right)=\int_{0}^{\infty} \int_{x}^{\infty} x^{r} y^{s} f_{m, n}(x, y) \mathrm{d} y \mathrm{~d} x, \\
0<x<y<\infty, \quad m=1,2, \ldots, \quad m<n, \quad r, s=0,1,2, \ldots .
\end{gathered}
$$

For the Lindley distribution, we obtain

$$
\begin{aligned}
\mu_{m, n}^{(r, s)}= & \frac{\theta^{4}}{\Gamma(m) \Gamma(n-m)(\theta+1)} \int_{0}^{\infty} \int_{x}^{\infty} x^{r} y^{s}\left[\log \left(\frac{\theta+1+\theta x}{\theta+1+\theta y}\right)+\theta(y-x)\right]^{n-m-1} \\
& \times\left\{-\log \left(\frac{\theta+1+\theta x}{\theta+1}\right)+\theta x\right\}^{m-1}(1+x)(1+y) \frac{e^{-\theta y}}{\theta+1+\theta x} \mathrm{~d} y \mathrm{~d} x .
\end{aligned}
$$

In general, the double moment in Eq. (4) cannot be obtained in a closed form, but for the special case when $n=m+1$, we can obtain a simplified closed form (see Appendix A).

Through numerical integration, we can determine the means, $\mu_{n}$, variances $\sigma_{n}^{2}=\mu_{n}^{(2)}-\mu_{n}^{2}(n=1,2, \ldots)$, and covariances $\sigma_{m, n}=\mu_{m, n}-$ $\mu_{m} \mu_{n}(m<n)$; of record statistics, respectively. Table 1 presents the values of $\mu_{n}$ for $n=1$ (1) 6 for different values of the shape parameter $\theta$. Table 2 provides the variances and covariances of record statistics for $m=1$ (1) $6 ; n=m+1$ (1) 6 and $\theta=0.5$ (0.5) 4.5 . The values of means, variances and covariances for $n \geq 7$ are computed but not presented here. All the computations were done by using Maple 16 . It may be observed from Table 1 , that the mean decreases when $\theta$ increases, also it increases when $n$ increases. From Table 2 , the variance and covariance decrease when $\theta$ increases. Also, the variances increase when $n$ increases. 
Table $1 \mid$ Means of record statistics.

\begin{tabular}{llllllllll}
\hline$n$ & $\theta=0.5$ & $\theta=1$ & $\theta=1.5$ & $\theta=2$ & $\theta=2.5$ & $\theta=3$ & $\theta=3.5$ & $\theta=4$ & $\theta=4.5$ \\
\hline 1 & 3.333 & 1.500 & 0.933 & 0.666 & 0.514 & 0.416 & 0.349 & 0.300 & 0.262 \\
2 & 6.069 & 2.819 & 1.785 & 1.289 & 1.002 & 0.816 & 0.686 & 0.591 & 0.518 \\
3 & 8.577 & 4.052 & 2.594 & 1.886 & 1.473 & 1.204 & 1.015 & 0.876 \\
4 & 10.969 & 5.236 & 3.375 & 2.466 & 1.933 & 1.584 & 1.338 & 1.157 & 1.018 \\
5 & 13.288 & 6.389 & 4.138 & 3.034 & 2.384 & 1.957 & 1.657 & 1.434 & 1.263 \\
6 & 15.559 & 7.520 & 4.888 & 3.594 & 2.830 & 2.327 & 1.972 & 1.709 & 1.507 \\
\hline
\end{tabular}

Table 2 Variances and covariances of record statistics.

\begin{tabular}{|c|c|c|c|c|c|c|c|c|c|c|}
\hline$m$ & $n$ & $\theta=0.5$ & $\theta=1$ & $\theta=1.5$ & $\theta=2$ & $\theta=2.5$ & $\theta=3$ & $\theta=3.5$ & $\theta=4$ & $\theta=4.5$ \\
\hline 1 & 1 & 7.5556 & 1.7500 & 0.7288 & 0.3888 & 0.2383 & 0.1597 & 0.1138 & 0.0850 & 0.0657 \\
\hline 1 & 2 & 6.8964 & 1.6323 & 0.6904 & 0.3724 & 0.2300 & 0.1550 & 0.1110 & 0.0832 & 0.0645 \\
\hline 1 & 3 & 6.5612 & 1.5655 & 0.6668 & 0.3616 & 0.2244 & 0.1518 & 0.1090 & 0.0818 & 0.0635 \\
\hline 1 & 4 & 6.3579 & 1.5227 & 0.6509 & 0.3541 & 0.2203 & 0.1493 & 0.1075 & 0.0808 & 0.0628 \\
\hline 1 & 5 & 6.2208 & 1.4929 & 0.6395 & 0.3486 & 0.2172 & 0.1475 & 0.1062 & 0.0800 & 0.0628 \\
\hline 1 & 6 & 6.1216 & 1.4709 & 0.6309 & 0.3444 & 0.2148 & 0.1460 & 0.1053 & 0.0793 & 0.0618 \\
\hline 2 & 2 & 12.639 & 3.0513 & 1.3096 & 0.7138 & 0.4444 & 0.3012 & 0.2167 & 0.1629 & 0.1266 \\
\hline 2 & 3 & 12.027 & 2.9267 & 1.2648 & 0.6932 & 0.4334 & 0.2949 & 0.2127 & 0.1603 & 0.1248 \\
\hline 2 & 4 & 11.656 & 2.8467 & 1.2346 & 0.6788 & 0.4256 & 0.2902 & 0.2097 & 0.1582 & 0.1234 \\
\hline 2 & 5 & 11.406 & 2.7910 & 1.2130 & 0.6682 & 0.4196 & 0.2865 & 0.2074 & 0.1566 & 0.1223 \\
\hline 2 & 6 & 11.225 & 2.7500 & 1.1967 & 0.6600 & 0.4150 & 0.2836 & 0.2055 & 0.1553 & 0.1213 \\
\hline 3 & 3 & 17.192 & 4.2143 & 1.8333 & 1.0102 & 0.6344 & 0.4331 & 0.3133 & 0.2366 & 0.1846 \\
\hline 3 & 4 & 16.663 & 4.0993 & 1.7896 & 0.9892 & 0.6228 & 0.4261 & 0.3089 & 0.2336 & 0.1825 \\
\hline 3 & 5 & 16.306 & 4.0193 & 1.7583 & 0.9737 & 0.6142 & 0.4208 & 0.3054 & 0.2312 & 0.1808 \\
\hline 3 & 6 & 16.047 & 3.9603 & 1.7347 & 0.9619 & 0.6074 & 0.4166 & 0.3026 & 0.2293 & 0.1794 \\
\hline 4 & 4 & 21.546 & 5.3189 & 2.3300 & 1.2919 & 0.8155 & 0.5592 & 0.4060 & 0.3075 & 0.2406 \\
\hline 4 & 5 & 21.085 & 5.2152 & 2.2893 & 1.2717 & 0.8037 & 0.5519 & 0.4012 & 0.3042 & 0.2384 \\
\hline 4 & 6 & 20.752 & 5.1387 & 2.2586 & 1.2562 & 0.7952 & 0.5466 & 0.3978 & 0.3019 & 0.2366 \\
\hline 5 & 5 & 25.800 & 6.3933 & 2.8120 & 1.5650 & 0.9912 & 0.6816 & 0.4962 & 0.3767 & 0.2952 \\
\hline 5 & 6 & 25.393 & 6.2996 & 2.7744 & 1.5460 & 0.9803 & 0.6742 & 0.4913 & 0.3733 & 0.2927 \\
\hline 6 & 6 & 29.994 & 7.4496 & 3.2850 & 1.8327 & 1.1635 & 0.8017 & 0.5846 & 0.4445 & 0.3489 \\
\hline
\end{tabular}

\section{LINEAR ESTIMATORS}

Let $Y_{U(1)}, Y_{U(2)} \cdots, Y_{U(n)}$ be the first $n$ upper record values from the three parameter Lindley distribution with pdf

$$
f_{Y}(y)=\frac{\theta^{2}}{\sigma^{2}(\theta+1)}(\sigma+y-\mu) e^{-\theta\left(\frac{y-\mu}{\sigma}\right)}, \quad y>\mu, \sigma>0, \theta>0,
$$

and let $X_{U(i)}=\frac{Y_{U(i)}-\mu}{\sigma}, i=, 1,2, \cdots, n$ be the corresponding upper record values from the standard Lindley distribution with pdf given in Eq. (2). Following the generalized least-squares approach, the BLUEs of $\mu$ and $\sigma$ can be derived as [29]

$$
\hat{\mu}_{B L U}=\sum_{i=1}^{n} a_{i} Y_{U(i)}, \quad \hat{\sigma}_{B L U}=\sum_{i=1}^{n} b_{i} Y_{U(i)},
$$

where

$$
\mathbf{a}=\left(a_{1}, \cdots, a_{n}\right)=\frac{\alpha^{\prime} \beta^{-1} \alpha \mathbf{1}^{\prime} \beta^{-1}-\alpha^{\prime} \beta^{-1} \mathbf{1} \alpha^{\prime} \beta^{-1}}{\left(\alpha^{\prime} \beta^{-1} \alpha\right)\left(\mathbf{1}^{\prime} \beta^{-1} \mathbf{1}\right)-\left(\alpha^{\prime} \beta^{-1} \mathbf{1}\right)^{2}},
$$

and

$$
\mathbf{b}=\left(b_{1}, \cdots, b_{n}\right)=\frac{\mathbf{1}^{\prime} \beta^{-1} \mathbf{1} \alpha^{\prime} \beta^{-1}-\mathbf{1}^{\prime} \beta^{-1} \alpha \mathbf{1}^{\prime} \beta^{-1}}{\left(\alpha^{\prime} \beta^{-1} \alpha\right)\left(\mathbf{1}^{\prime} \beta^{-1} \mathbf{1}\right)-\left(\alpha^{\prime} \beta^{-1} \mathbf{1}\right)^{2}},
$$


$\alpha^{\prime}=\left(\alpha_{1}, \alpha_{2}, \cdots, \alpha_{n}\right)$ is the moment vector with $\alpha_{i}=E\left(X_{U(i)}\right)$ and $\beta=\left(\beta_{i, j}\right), 1 \leq i \leq j \leq n$ is the covariance matrix with $\beta_{i, j}=$ $\operatorname{Cov}\left(X_{U(i)}, X_{U(j)}\right)$, and $\mathbf{1}^{\prime}=(1,1, \cdots, 1)_{1 \times n}$.

Furthermore, the variances of these BLUEs are given by

$$
\begin{aligned}
& \operatorname{Var}\left(\hat{\mu}_{B L U}\right)=\left[\frac{\alpha^{\prime} \beta^{-1} \alpha}{\left(\alpha^{\prime} \beta^{-1} \alpha\right)\left(\mathbf{1}^{\prime} \beta^{-1} \mathbf{1}\right)-\left(\alpha^{\prime} \beta^{-1} \mathbf{1}\right)^{2}}\right] \sigma^{2}=V_{1} \sigma^{2}, \\
& \operatorname{Var}\left(\hat{\sigma}_{B L U}\right)=\left[\frac{\mathbf{1}^{\prime} \beta^{-1} \mathbf{1}}{\left(\alpha^{\prime} \beta^{-1} \alpha\right)\left(\mathbf{1}^{\prime} \beta^{-1} \mathbf{1}\right)-\left(\alpha^{\prime} \beta^{-1} \mathbf{1}\right)^{2}}\right] \sigma^{2}=V_{2} \sigma^{2},
\end{aligned}
$$

and

$$
\operatorname{Cov}\left(\hat{\mu}_{B L U}, \hat{\sigma}_{B L U}\right)=\left[\frac{-\alpha^{\prime} \beta^{-1} \mathbf{1}}{\left(\alpha^{\prime} \beta^{-1} \alpha\right)\left(\mathbf{1}^{\prime} \beta^{-1} \mathbf{1}\right)-\left(\alpha^{\prime} \beta^{-1} \mathbf{1}\right)^{2}}\right] \sigma^{2}=V_{3} \sigma^{2}
$$

The coefficients $a_{i}$ 's, $b_{i}$ 's, $1 \leq i \leq n$ and the values of $V_{1}, V_{2}$ and $V_{3}$ are computed and presented in Tables 3-5, respectively. From Table 5, we note that the variances of the BLUEs decrease as $n$ increases.

Based on the BLUEs of the location and scale parameters, the CIs for $\mu$ and $\sigma$ can be constructed through the pivotal quantities given by

$$
R_{1}=\frac{\hat{\mu}_{B L U}-\mu}{\hat{\sigma}_{B L U} \sqrt{V_{1}}} \text { and } R_{2}=\frac{\hat{\sigma}_{B L U}-\sigma}{\sigma \sqrt{V_{2}}}
$$

Constructing such CIs requires then percentage the points of $R_{1}$ and $R_{2}$ which can be computed by using the BLUEs $\hat{\mu}_{B L U}$ and $\hat{\sigma}_{B L U}$ via Monte Carlo method. In Table 6, we have determined the percentage points of $R_{1}$ and $R_{2}$ based on 10000 runs and different values of $n$ and $\theta$. Based on these simulated percentage points, we can determine a $100(1-\alpha) \%$ CI for $\mu$ through the pivotal quantity $R_{1}$ as follows

$$
P\left(\hat{\mu}_{B L U}-\hat{\sigma}_{B L U} \sqrt{V_{1}} R_{1}(1-\alpha / 2) \leq \mu \leq \hat{\mu}_{B L U}-\hat{\sigma}_{B L U} \sqrt{V_{1}} R_{1}(\alpha / 2)\right)=1-\alpha,
$$

where $R_{1}(\gamma)$ is the left percentage point of $R_{1}$ at $\gamma$, i.e., $P\left(R_{1}<R_{1}(\gamma)\right)=\gamma$.

Similarly, a $100(1-\alpha) \%$ CI for $\sigma$ can be constructed through the pivotal quantity $R_{2}$ as follows

$$
P\left(\frac{\hat{\sigma}_{B L U}}{1+\sqrt{V_{2}} R_{2}(1-\alpha / 2)} \leq \sigma \leq \frac{\hat{\sigma}_{B L U}}{1+\sqrt{V_{2}} R_{2}(\alpha / 2)}\right)=1-\alpha .
$$

Now, let us consider the BLIEs of $\mu$ and $\sigma$. Based on the results of Mann [30], the BLIEs for $\mu$ and $\sigma$ are (see also [4], p. 143)

$$
\hat{\mu}_{B L I}=\hat{\mu}_{B L U}-\frac{V_{3}}{1+V_{2}} \hat{\sigma}_{B L U} \quad \text { and } \quad \hat{\sigma}_{B L I}=\frac{\hat{\sigma}_{B L U}}{1+V_{2}},
$$

Table 3 Coefficients for the BLUEs of $\mu$.

\begin{tabular}{llllllllll}
\hline$n$ & $\theta=0.5$ & $\theta=1$ & $\theta=1.5$ & $\theta=2$ & $\theta=2.5$ & $\theta=3$ & $\theta=3.5$ & $\theta=4$ & $\theta=4.5$ \\
\hline 2 & 2.2184 & 2.1369 & 2.0949 & 2.0698 & 2.0536 & 2.0428 & 2.0352 & 2.0295 & 2.0245 \\
& -1.2184 & -1.1369 & -1.0949 & -1.0698 & -1.0536 & -1.0428 & -1.0352 & -1.0295 & -1.0245 \\
\hline 3 & 1.6086 & 1.5680 & 1.5472 & 1.5351 & 1.5268 & 1.5207 & 1.5173 & 1.5148 & 1.5133 \\
& 0.0564 & 0.0404 & 0.0310 & 0.0228 & 0.0188 & 0.0168 & 0.0133 & 0.0106 & 0.0074 \\
& -0.6650 & -0.6085 & -0.5774 & -0.5580 & -0.5456 & -0.5375 & -0.5307 & -0.5254 & -0.5207 \\
\hline 4 & 1.3957 & 1.3721 & 1.3604 & 1.3536 & 1.3487 & 1.3452 & 1.3433 & 1.3421 & 1.3410 \\
& 0.0526 & 0.0367 & 0.0267 & 0.0198 & 0.0165 & 0.0146 & 0.0109 & 0.0077 & 0.0059 \\
& 0.0223 & 0.0173 & 0.0136 & 0.0113 & 0.0086 & 0.0060 & 0.0070 & 0.0078 & 0.0073 \\
& -0.4707 & -0.4262 & -0.4008 & -0.3848 & -0.3739 & -0.3659 & -0.3613 & -0.3577 & -0.3543 \\
\hline 5 & 1.2850 & 1.2710 & 1.2646 & 1.2611 & 1.2585 & 1.2563 & 1.2556 & 1.2550 \\
& 0.0506 & 0.0347 & 0.0250 & 0.0183 & 0.0149 & 0.0128 & 0.0105 & 0.0070 & 0.0030 \\
& 0.0223 & 0.0171 & 0.0132 & 0.0107 & 0.0090 & 0.0065 & 0.0067 & 0.0074 & 0.0062 \\
& 0.0108 & 0.0090 & 0.0075 & 0.0064 & 0.0040 & 0.0047 & 0.0009 & 0.0017 & 0.0151 \\
& -0.3688 & -0.3320 & -0.3104 & -0.2967 & -0.2865 & -0.2803 & -0.2737 & -0.2712 & -0.2776 \\
& & & & & & &
\end{tabular}


Table 4 Coefficients for the BLUEs of $\sigma$.

\begin{tabular}{llllllllll}
\hline$n$ & $\theta=0.5$ & $\theta=1$ & $\theta=1.5$ & $\theta=2$ & $\theta=2.5$ & $\theta=3$ & $\theta=3.5$ & $\theta=4$ & $\theta=4.5$ \\
\hline 2 & -0.3655 & -0.7579 & -1.1731 & -1.6048 & -2.0491 & -2.5031 & -2.9647 & -3.4317 & -3.9016 \\
& 0.3655 & 0.7579 & 1.1731 & 1.6048 & 2.0491 & 2.5031 & 2.9647 & 3.4317 & 3.9016 \\
3 & -0.1889 & -0.3881 & -0.5968 & -0.8131 & -1.0352 & -1.2608 & -1.4910 & -1.7236 & -1.9602 \\
& -0.0036 & -0.0074 & -0.0107 & -0.0131 & -0.0151 & -0.0183 & -0.0187 & -0.0203 & -0.0174 \\
& 0.1925 & 0.3956 & 0.6076 & 0.8263 & 1.0503 & 1.2791 & 1.5098 & 1.7439 & 1.9776 \\
\hline 4 & -0.1292 & -0.2639 & -0.4041 & -0.5486 & -0.6964 & -0.8466 & -1.0002 & -1.1554 & -1.3115 \\
& -0.0025 & -0.0051 & -0.0072 & -0.0088 & -0.0109 & -0.0132 & -0.0119 & -0.0110 & -0.0119 \\
& -0.0002 & -0.0009 & -0.0020 & -0.0032 & -0.0037 & -0.0036 & -0.0071 & -0.0099 & -0.0101 \\
& 0.1320 & 0.2700 & 0.4134 & 0.5607 & 0.7111 & 0.8636 & 1.0194 & 1.1764 & 1.3335 \\
\hline 5 & -0.0989 & -0.2013 & -0.3071 & -0.4159 & -0.5269 & -0.6395 & -0.7540 & -0.8711 & -0.9915 \\
& -0.0020 & -0.0039 & -0.0055 & -0.0067 & -0.0077 & -0.0089 & -0.0106 & -0.0085 & -0.0012 \\
& -0.0002 & -0.0007 & -0.0015 & -0.0023 & -0.0045 & -0.0047 & -0.0063 & -0.0087 & -0.0060 \\
& 0.0003 & 0.0003 & -0.00004 & -0.0006 & 0.0011 & 0.0001 & 0.0026 & 0.0025 & -0.0137 \\
& 0.1008 & 0.2057 & 0.3143 & 0.4257 & 0.5381 & 0.6530 & 0.7684 & 0.8858 & 1.0125 \\
\hline
\end{tabular}

Table 5 Variances and covariances of the BLUEs of $\mu$ and $\sigma$ in terms of $\sigma^{2}$ and $V_{4}$.

\begin{tabular}{|c|c|c|c|c|c|c|c|c|c|}
\hline$n$ & $\theta=0.5$ & $\theta=1$ & $\theta=1.5$ & $\theta=2$ & $\theta=2.5$ & $\theta=3$ & $\theta=3.5$ & $\theta=4$ & $\theta=4.5$ \\
\hline \multirow[t]{4}{*}{2} & 18.6661 & 4.0041 & 1.6012 & 0.8333 & 0.5030 & 0.3335 & 0.2358 & 0.1750 & 0.1068 \\
\hline & 0.8553 & 0.8828 & 0.9050 & 0.9215 & 0.9351 & 0.9454 & 0.9536 & 0.9597 & 0.9636 \\
\hline & -3.0922 & -1.4135 & -0.8897 & -0.6406 & -0.4978 & -0.4056 & -0.3413 & -0.2941 & -0.2577 \\
\hline & 2.2462 & 1.1335 & 0.7385 & 0.5434 & 0.4292 & 0.3537 & 0.3005 & 0.2592 & 0.2277 \\
\hline \multirow[t]{4}{*}{3} & 13.5075 & 2.9438 & 1.1861 & 0.6201 & 0.3750 & 0.2489 & 0.1762 & 0.1310 & 0.1009 \\
\hline & 0.4226 & 0.4347 & 0.4453 & 0.4539 & 0.4607 & 0.4663 & 0.4706 & 0.4748 & 0.4779 \\
\hline & -1.5981 & -0.7242 & -0.4529 & -0.3248 & -0.2514 & -0.2042 & -0.1715 & -0.1480 & -0.1298 \\
\hline & 1.0724 & 0.5430 & 0.3581 & 0.2660 & 0.2109 & 0.1743 & 0.1487 & 0.1297 & 0.1147 \\
\hline \multirow[t]{4}{*}{4} & 11.7063 & 2.5784 & 1.0444 & 0.5476 & 0.3318 & 0.2206 & 0.1561 & 0.1161 & 0.0895 \\
\hline & 0.2810 & 0.2880 & 0.2946 & 0.3000 & 0.3045 & 0.3083 & 0.3112 & 0.3139 & 0.3161 \\
\hline & -1.0929 & -0.4927 & -0.3067 & -0.2192 & -0.1692 & -0.1373 & -0.1150 & -0.0990 & -0.0868 \\
\hline & 0.6940 & 0.3516 & 0.2323 & 0.1743 & 0.1390 & 0.1156 & 0.0981 & 0.0860 & 0.0801 \\
\hline \multirow[t]{4}{*}{5} & 10.7697 & 2.3899 & 0.9718 & 0.5107 & 0.3099 & 0.2061 & 0.1461 & 0.1087 & 0.0833 \\
\hline & 0.2110 & 0.2157 & 0.2201 & 0.2240 & 0.2272 & 0.2298 & 0.2323 & 0.2342 & 0.2347 \\
\hline & -0.8369 & -0.3759 & -0.2332 & -0.1662 & -0.1281 & -0.1037 & -0.0869 & -0.0746 & -0.0645 \\
\hline & 0.5101 & 0.2584 & 0.1733 & 0.1290 & 0.1029 & 0.0858 & 0.0737 & 0.0640 & 0.0537 \\
\hline
\end{tabular}

Note: For each $n(n=2, \cdots, 5)$, the first, second, third and the fourth lines represent $V_{1}=\frac{1}{\sigma^{2}} \operatorname{Var}\left(\hat{\mu}_{B L U}\right), V_{2}=\frac{1}{\sigma^{2}} \operatorname{Var}\left(\hat{\sigma}_{B L U}\right), V_{3}=\frac{1}{\sigma^{2}} \operatorname{Cov}\left(\hat{\mu}_{B L U}, \hat{\sigma}_{B L U}\right)$ and $V_{4}$, respectively.

where $V_{1}=\frac{1}{\sigma^{2}} \operatorname{Var}\left(\hat{\mu}_{B L U}\right), V_{2}=\frac{1}{\sigma^{2}} \operatorname{Var}\left(\hat{\sigma}_{B L U}\right)$ and

$$
V_{3}=\frac{1}{\sigma^{2}} \operatorname{Cov}\left(\hat{\mu}_{B L U}, \hat{\sigma}_{B L U}\right)
$$

Furthermore the variances of these BLIEs are given by (see Arnold et al. [4], p. 143)

$$
\operatorname{Var}\left(\hat{\mu}_{B L I}\right)=\sigma^{2}\left(V_{1}-\frac{V_{3}^{2}\left(2+V_{2}\right)}{\left(1+V_{2}\right)^{2}}\right) \quad \text { and } \operatorname{Var}\left(\hat{\sigma}_{B L I}\right)=\frac{\sigma^{2} V_{2}}{\left(1+V_{2}\right)^{2}}
$$


Table 6 Simulated percentage points of $R_{1}$ and $R_{2}$.

\begin{tabular}{|c|c|c|c|c|c|c|c|c|c|}
\hline \multirow[b]{2}{*}{$\theta$} & \multirow[b]{2}{*}{$n$} & \multicolumn{4}{|c|}{$R_{1}$} & \multicolumn{4}{|c|}{$R_{2}$} \\
\hline & & $2.5 \%$ & $5 \%$ & $95 \%$ & $97.5 \%$ & $2.5 \%$ & $5 \%$ & $95 \%$ & $97.5 \%$ \\
\hline \multirow[t]{4}{*}{0.5} & 2 & -0.7440 & -0.7155 & 14.492 & 26.198 & -1.0478 & -1.0213 & 1.9622 & 2.6028 \\
\hline & 3 & -0.8658 & -0.8346 & 4.8155 & 7.6618 & -1.3099 & -1.2264 & 1.9176 & 2.4617 \\
\hline & 4 & -0.9355 & -0.9016 & 3.9282 & 5.5012 & -1.4486 & -1.3088 & 1.8902 & 2.3525 \\
\hline & 5 & -0.9773 & -0.9332 & 3.2299 & 4.5157 & -1.5113 & -1.3567 & 1.8260 & 2.3621 \\
\hline \multirow[t]{4}{*}{1} & 2 & -0.7280 & -0.7038 & 13.305 & 30.067 & -1.0378 & -1.0041 & 1.9768 & 2.5454 \\
\hline & 3 & -0.8464 & -0.8227 & 5.2436 & 7.8190 & -1.3088 & -1.2210 & 1.8889 & 2.4898 \\
\hline & 4 & -0.9030 & -0.8733 & 3.6417 & 5.4939 & -1.4414 & -1.2896 & 1.8967 & 2.3361 \\
\hline & 5 & -0.9404 & -0.9018 & 3.1786 & 4.7170 & -1.5085 & -1.3516 & 1.8264 & 2.2896 \\
\hline \multirow[t]{4}{*}{1.5} & 2 & -0.7174 & -0.6948 & 13.769 & 25.300 & -1.0205 & -0.9960 & 1.9763 & 2.6424 \\
\hline & 3 & -0.8291 & -0.8042 & 4.6772 & 7.4613 & -1.2891 & -1.2059 & 1.9158 & 2.4790 \\
\hline & 4 & -0.8848 & -0.8631 & 3.8001 & 5.5215 & -1.4325 & -1.3015 & 1.8816 & 2.3670 \\
\hline & 5 & -0.9198 & -0.8902 & 3.1424 & 4.5661 & -1.4954 & -1.3501 & 1.8362 & 2.3643 \\
\hline \multirow[t]{4}{*}{2} & 2 & -0.7110 & -0.6905 & 12.911 & 29.571 & -1.0165 & -0.9825 & 1.9599 & 2.5509 \\
\hline & 3 & -0.8249 & -0.8003 & 4.8515 & 7.8426 & -1.2871 & -1.1963 & 1.9217 & 2.4887 \\
\hline & 4 & -0.8781 & -0.8474 & 3.8368 & 5.4495 & -1.4125 & -1.3013 & 1.8700 & 2.4487 \\
\hline & 5 & -0.9055 & -0.8821 & 3.3815 & 4.5120 & -1.4831 & -1.3580 & 1.8812 & 2.2959 \\
\hline \multirow[t]{4}{*}{2.5} & 2 & -0.7061 & -0.6846 & 13.532 & 24.754 & -1.0049 & -0.9816 & 1.9843 & 2.6678 \\
\hline & 3 & -0.8142 & -0.7911 & 4.6038 & 7.3439 & -1.2746 & -1.1912 & 1.9176 & 2.4850 \\
\hline & 4 & -0.8689 & -0.8476 & 3.7523 & 5.3652 & -1.4201 & -1.2933 & 1.8914 & 2.3739 \\
\hline & 5 & -0.8992 & -0.8737 & 3.0930 & 4.5540 & -1.4836 & -1.3446 & 1.8430 & 2.3753 \\
\hline \multirow[t]{4}{*}{3} & 2 & -0.7042 & -0.6832 & 12.743 & 26.604 & -1.0038 & -0.9713 & 1.9557 & 2.5370 \\
\hline & 3 & -0.8134 & -0.7910 & 4.7970 & 7.5683 & -1.2730 & -1.1860 & 1.9253 & 2.4916 \\
\hline & 4 & -0.8659 & -0.8354 & 3.7999 & 5.4028 & -1.4223 & -1.2896 & 1.8683 & 2.4701 \\
\hline & 5 & -0.8927 & -0.8694 & 3.3642 & 4.5019 & -1.4798 & -1.3543 & 1.8802 & 2.3110 \\
\hline \multirow[t]{4}{*}{3.5} & 2 & -0.7006 & -0.6797 & 11.786 & 24.528 & -0.9955 & -0.9666 & 2.0320 & 2.6852 \\
\hline & 3 & -0.8074 & -0.7856 & 4.6534 & 7.2653 & -1.2652 & -1.1790 & 1.9438 & 2.4921 \\
\hline & 4 & -0.8627 & -0.8371 & 3.7762 & 5.2676 & -1.4081 & -1.2911 & 1.9073 & 2.3811 \\
\hline & 5 & -0.8905 & -0.8639 & 3.1165 & 4.5332 & -1.4773 & -1.3310 & 1.8715 & 2.3824 \\
\hline \multirow[t]{4}{*}{4} & 2 & -0.6986 & -0.6781 & 13.615 & 28.989 & -0.9966 & -0.9713 & 1.9175 & 2.5425 \\
\hline & 3 & -0.8077 & -0.7839 & 5.1292 & 7.5166 & -1.2635 & -1.1831 & 1.9524 & 2.4930 \\
\hline & 4 & -0.8590 & -0.8376 & 3.5764 & 5.3673 & -1.3959 & -1.2822 & 1.9260 & 2.4645 \\
\hline & 5 & -0.8863 & -0.8140 & 1.9541 & 4.4724 & -1.4698 & -1.3271 & 1.8644 & 2.3214 \\
\hline \multirow[t]{4}{*}{4.5} & 2 & -0.6974 & -0.6768 & 11.708 & 24.395 & -0.9905 & -0.9620 & 2.0339 & 2.6996 \\
\hline & 3 & -0.8029 & -0.7804 & 4.6256 & 7.2221 & -1.2576 & -1.1724 & 1.9455 & 2.4970 \\
\hline & 4 & -0.8561 & -0.8314 & 3.7530 & 5.3086 & -1.3999 & -1.2862 & 1.9036 & 2.3828 \\
\hline & 5 & -0.8866 & -0.8609 & 3.1101 & 4.5284 & -1.4740 & -1.3323 & 1.8860 & 2.3939 \\
\hline
\end{tabular}

Based on the BLIEs, we can again construct CIs for the location and scale parameters through pivotal quantities given by

$$
R_{3}=\frac{\hat{\mu}_{B L I}-\mu}{\hat{\sigma}_{B L I} \sqrt{V_{1}-\frac{V_{3}^{2}\left(2+V_{2}\right)}{\left(1+V_{2}\right)^{2}}}} \text { and } R_{4}=\frac{\hat{\sigma}_{B L I}-\sigma}{\sigma \frac{\sqrt{V_{2}}}{1+V_{2}}} .
$$

Table 7 presents the percentage points of $R_{3}$ and $R_{4}$ based on 10000 runs and different choices of $n$ and $\theta$. With the BLIEs and the use of Table 7 , we can determine a $100(1-\alpha) \%$ CI for $\mu$ through the pivotal quantity $R_{3}$ as

$$
P\left(\hat{\mu}_{B L I}-\hat{\sigma}_{B L I} \sqrt{V_{1}-\frac{V_{3}^{2}\left(2+V_{2}\right)}{\left(1+V_{2}\right)^{2}}} R_{3}(1-\alpha / 2) \leq \mu \leq \hat{\mu}_{B L I}-\hat{\sigma}_{B L I} \sqrt{V_{1}-\frac{V_{3}^{2}\left(2+V_{2}\right)}{\left(1+V_{2}\right)^{2}}} R_{3}(\alpha / 2)\right)=1-\alpha,
$$


Table 7 Simulated percentage points of $R_{3}$ and $R_{4}$.

\begin{tabular}{|c|c|c|c|c|c|c|c|c|c|}
\hline \multirow[b]{2}{*}{$\theta$} & \multirow[b]{2}{*}{$n$} & \multicolumn{4}{|c|}{$R_{3}$} & \multicolumn{4}{|c|}{$R_{4}$} \\
\hline & & $2.5 \%$ & $5 \%$ & $95 \%$ & $97.5 \%$ & $2.5 \%$ & $5 \%$ & $95 \%$ & $97.5 \%$ \\
\hline \multirow[t]{4}{*}{0.5} & 2 & -0.7192 & -0.6654 & 28.847 & 62.296 & -1.9750 & -1.9440 & 1.0862 & 1.7186 \\
\hline & 3 & -0.8521 & -0.8038 & 8.1365 & 12.851 & -1.9613 & -1.8648 & 1.3133 & 1.8644 \\
\hline & 4 & -0.9076 & -0.8575 & 5.3955 & 8.0337 & -1.9595 & -1.8304 & 1.3756 & 1.8680 \\
\hline & 5 & -0.9494 & -0.8981 & 4.1167 & 5.8680 & -1.9598 & -1.8096 & 1.4121 & 1.8921 \\
\hline \multirow[t]{4}{*}{1} & 2 & -0.7166 & -0.6676 & 29.839 & 61.833 & -1.9773 & -1.9486 & 0.9652 & 1.6058 \\
\hline & 3 & -0.8298 & -0.7854 & 8.4338 & 12.824 & -1.9744 & -1.8783 & 1.2793 & 1.7441 \\
\hline & 4 & -0.8847 & -0.8414 & 5.33826 & 7.7013 & -1.9625 & -1.8453 & 1.3580 & 1.8268 \\
\hline & 5 & -0.9195 & -0.8769 & 4.25628 & 6.0973 & -1.9563 & -1.8040 & 1.3949 & 1.8661 \\
\hline \multirow[t]{4}{*}{1.5} & 2 & -0.7145 & -0.6753 & 27.652 & 55.661 & -1.9727 & -1.9445 & 1.0212 & 1.6648 \\
\hline & 3 & -0.8232 & -0.7870 & 8.0750 & 12.324 & -1.9601 & -1.8716 & 1.2314 & 1.7524 \\
\hline & 4 & -0.8707 & -0.8317 & 5.1743 & 7.9021 & -1.9593 & -1.8315 & 1.3280 & 1.8161 \\
\hline & 5 & -0.9036 & -0.8643 & 4.2503 & 5.9628 & -1.9602 & -1.7976 & 1.4296 & 1.9293 \\
\hline \multirow[t]{4}{*}{2} & 2 & -0.7137 & -0.6689 & 29.571 & 61.791 & -1.9764 & -1.9493 & 0.9459 & 1.5909 \\
\hline & 3 & -0.8188 & -0.7820 & 7.8548 & 12.669 & -1.9664 & -1.8770 & 1.2148 & 1.7904 \\
\hline & 4 & -0.8659 & -0.8324 & 5.2732 & 7.5969 & -1.9651 & -1.8405 & 1.3254 & 1.8318 \\
\hline & 5 & -0.8971 & -0.8627 & 4.1960 & 5.8944 & -1.9663 & -1.8263 & 1.4045 & 1.8859 \\
\hline \multirow[t]{4}{*}{2.5} & 2 & -0.7103 & -0.6684 & 28.695 & 56.244 & -1.9737 & -1.9464 & 1.0746 & 1.6639 \\
\hline & 3 & -0.8173 & -0.7803 & 7.5279 & 12.031 & -1.9717 & -1.8797 & 1.2505 & 1.7248 \\
\hline & 4 & -0.8637 & -0.8334 & 5.2897 & 7.9068 & -1.9700 & -1.8504 & 1.2999 & 1.7596 \\
\hline & 5 & -0.8874 & -0.8573 & 4.2208 & 5.9416 & -1.9688 & -1.8205 & 1.3881 & 1.8779 \\
\hline \multirow[t]{4}{*}{3} & 2 & -0.7095 & -0.6700 & 27.772 & 59.816 & -1.9730 & -1.9452 & 0.9777 & 1.6110 \\
\hline & 3 & -0.8156 & -0.7806 & 7.8232 & 12.635 & -1.9624 & -1.8745 & 1.2103 & 1.7864 \\
\hline & 4 & -0.8567 & -0.8272 & 5.2928 & 7.7807 & -1.9664 & -1.8263 & 1.3073 & 1.8655 \\
\hline & 5 & -0.8878 & -0.8573 & 4.0903 & 5.9822 & -1.9646 & -1.8219 & 1.3738 & 1.9174 \\
\hline \multirow[t]{4}{*}{3.5} & 2 & -0.7119 & -0.6713 & 28.026 & 59.938 & -1.9737 & -1.9483 & 1.0317 & 1.7035 \\
\hline & 3 & -0.8122 & -0.7744 & 7.7792 & 12.530 & -1.9656 & -1.8776 & 1.2310 & 1.8115 \\
\hline & 4 & -0.8607 & -0.8278 & 5.2559 & 7.8655 & -1.9572 & -1.8424 & 1.3075 & 1.8556 \\
\hline & 5 & -0.8879 & -0.8564 & 4.1511 & 5.9445 & -1.9560 & -1.8153 & 1.4402 & 1.9335 \\
\hline \multirow[t]{4}{*}{4} & 2 & -0.7135 & -0.6704 & 29.338 & 61.613 & -1.9763 & -1.9510 & 0.9379 & 1.5628 \\
\hline & 3 & -0.8109 & -0.7820 & 7.9785 & 12.891 & -1.9664 & -1.8858 & 1.2750 & 1.8004 \\
\hline & 4 & -0.8568 & -0.8233 & 5.2535 & 7.6812 & -1.9626 & -1.8359 & 1.3215 & 1.8401 \\
\hline & 5 & -0.8831 & -0.8545 & 4.2507 & 5.9219 & -1.9621 & -1.8221 & 1.4286 & 1.9605 \\
\hline \multirow[t]{4}{*}{4.5} & 2 & -0.7128 & -0.6725 & 28.003 & 59.957 & -1.9737 & -1.9488 & 1.0271 & 1.6993 \\
\hline & 3 & -0.8113 & -0.7753 & 8.1742 & 12.527 & -1.9622 & -1.8771 & 1.2054 & 1.7388 \\
\hline & 4 & -0.8588 & -0.8283 & 5.0210 & 7.5207 & -1.9526 & -1.8453 & 1.3567 & 1.8113 \\
\hline & 5 & -0.8854 & -0.8554 & 4.0604 & 5.9901 & -1.9475 & -1.8141 & 1.3953 & 1.9071 \\
\hline
\end{tabular}

Similarly, we can determine a $100(1-\alpha) \% \mathrm{CI}$ for $\sigma$, through the pivotal quantity $R_{4}$ as

$$
P\left(\frac{\hat{\sigma}_{B L I}}{1+\frac{\sqrt{V_{2}}}{1+V_{2}} R_{4}(1-\alpha / 2)} \leq \sigma \leq \frac{\hat{\sigma}_{B L I}}{1+\frac{\sqrt{V_{2}}}{1+V_{2}} R_{4}(\alpha / 2)}\right)=1-\alpha .
$$

Now, let us compare the BLUEs and BLIEs using the relative efficiency criterion (REC). Since the mean squared errors (MSEs) of BLUEs are equal to their corresponding variances, we have

$$
\operatorname{MSE}\left(\hat{\mu}_{B L U}\right)=\sigma^{2} V_{1} \quad \text { and } \quad \operatorname{MSE}\left(\hat{\sigma}_{B L U}\right)=\sigma^{2} V_{2} .
$$


On the other hand, the MSEs of BLIEs of $\mu$ and $\sigma$ can be obtained as

$$
\operatorname{MSE}\left(\hat{\mu}_{B L I}\right)=\sigma^{2}\left(V_{1}-\frac{V_{3}^{2}}{1+V_{2}}\right) \text { and } \operatorname{MSE}\left(\hat{\sigma}_{B L I}\right)=\frac{\sigma^{2} V_{2}}{1+V_{2}} .
$$

Therefore, we can readily obtain the RECs of the BLIEs of $\mu$ and $\sigma$ with respect to their corresponding BLUEs as follows

$$
\operatorname{REC}\left(\hat{\mu}_{B L I}, \hat{\mu}_{B L U}\right)=\frac{\operatorname{MSE}\left(\hat{\mu}_{B L U}\right)}{\operatorname{MSE}\left(\hat{\mu}_{B L I}\right)}=\frac{V_{1}}{V_{1}-\frac{V_{3}^{2}}{1+V_{2}}} \geq 1, \operatorname{REC}\left(\hat{\sigma}_{B L I}, \hat{\sigma}_{B L U}\right)=\frac{\operatorname{MSE}\left(\hat{\sigma}_{B L U}\right)}{\operatorname{MSE}\left(\hat{\sigma}_{B L I}\right)}=1+V_{2} \geq 1 .
$$

Therefore, both of the BLIEs of $\mu$ and $\sigma$ perform better than the corresponding BLUEs in terms of MSEs.

\section{LINEAR PREDICTORS}

Suppose we have observed the first $n$ upper records $\mathbf{Y}^{\prime}=\left(Y_{U(1)}, Y_{U(2)} \cdots, Y_{U(n)}\right)$ from the three parameter Lindley distribution with pdf given in Eq. (5) and our aim is to predict the next upper record $Y=Y_{U(n+1)}$. The best linear unbiased predictor (BLUP) of $Y$ is given by (Arnold et al. [4], p. 150)

$$
\hat{Y}_{B L U P}=\hat{\mu}_{B L U}+\hat{\sigma}_{B L U} \alpha_{n+1}+\omega^{\prime} \beta^{-1}\left(\mathbf{Y}-\hat{\mu}_{B L U} 1-\hat{\sigma}_{B L U} \alpha\right),
$$

where

$$
\omega^{\prime}=\left(\operatorname{Cov}\left(X_{U(1)}, X_{U(n+1)}\right), \cdots, \operatorname{Cov}\left(X_{U(n)}, X_{U(n+1)}\right)\right)
$$

in which $X_{U(i)}=\frac{Y_{U(i)}-\mu}{\sigma}, i=, 1,2, \cdots, n+1$. Moreover, the mean squared prediction error (MSPE) of $\hat{Y}_{B L U P}$ is given by (Burkschat [31])

$$
\begin{aligned}
\operatorname{MSPE}\left(\hat{Y}_{\text {BLUP }}\right)= & E\left[\left(\hat{Y}_{B L U P}-Y_{U(n+1)}\right)^{2}\right] \\
= & \sigma^{2}\left[\left(1-\omega^{\prime} \beta^{-1} \mathbf{1}\right)^{2} V_{1}+\left(\alpha_{n+1}-\omega^{\prime} \beta^{-1} \alpha\right)^{2} V_{2}-\omega^{\prime} \beta^{-1} \omega\right. \\
& \left.+2\left(1-\omega^{\prime} \beta^{-1} \mathbf{1}\right)\left(\alpha_{n+1}-\omega^{\prime} \beta^{-1} \alpha\right) V_{3}+\operatorname{Var}\left(X_{U(n+1)}\right)\right] .
\end{aligned}
$$

Let us now consider the best linear invariant predictor (BLIP) of the next upper record value. From the results of Mann [30], the BLIP of $Y$ can be obtained based on the BLUP of $Y$ as follows (see also [4], p. 153)

$$
\hat{Y}_{B L I P}=\hat{Y}_{B L U P}-\left(\frac{V_{4}}{1+V_{2}}\right) \hat{\sigma}_{B L U},
$$

where $\hat{Y}_{B L U P}$ is the BLUP of $Y_{U(n+1)}$ and

$$
V_{4}=\left(1-\omega^{\prime} \beta^{-1} \mathbf{1}\right) V_{3}+\left(\alpha_{n+1}-\omega^{\prime} \beta^{-1} \alpha\right) V_{2} .
$$

In Table 5, we reported the values of $V_{4}$ for different values of $n$ and $\theta$.

The MSPE of $\hat{Y}_{\text {BLIP }}$ is given by (Burkschat [31])

$$
\begin{aligned}
\operatorname{MSPE}\left(\hat{Y}_{B L I P}\right)= & E\left[\left(\hat{Y}_{B L I P}-Y_{U(n+1)}\right)^{2}\right] \\
= & \sigma^{2}\left[\frac{\alpha^{\prime} \beta^{-1} \alpha+1}{\Delta}\left(1-\omega^{\prime} \beta^{-1} \mathbf{1}\right)^{2}-\omega^{\prime} \beta^{-1} \omega+\frac{\mathbf{1}^{\prime} \beta^{-1} \mathbf{1}}{\Delta}\left(\alpha_{n+1}-\omega^{\prime} \beta^{-1} \alpha\right)^{2}\right. \\
& \left.+\operatorname{Var}\left(X_{U(n+1)}\right)-2 \frac{\alpha^{\prime} \beta^{-1} \mathbf{1}}{\Delta}\left(1-\omega^{\prime} \beta^{-1} \mathbf{1}\right)\left(\alpha_{n+1}-\omega^{\prime} \beta^{-1} \alpha\right)\right],
\end{aligned}
$$

where

$$
\Delta=\left(\alpha^{\prime} \beta^{-1} \alpha+1\right)\left(\mathbf{1}^{\prime} \beta^{-1} \mathbf{1}\right)-\left(\alpha^{\prime} \beta^{-1} \mathbf{1}\right)^{2}
$$

Now we compare the BLUP and BLIP of $Y$ using the REC. The REC of $\hat{Y}_{B L I P}$ relative to $\hat{Y}_{B L U P}$ is

$$
\operatorname{REC}\left(\hat{Y}_{B L I P}, \hat{Y}_{B L U P}\right)=\frac{\operatorname{MSPE}\left(\hat{Y}_{B L U P}\right)}{\operatorname{MSPE}\left(\hat{Y}_{B L I P}\right)} .
$$


Table 8 The REC of $\hat{Y}_{B L I P}$ with respect to $\hat{Y}_{B L U P}$.

\begin{tabular}{llllllllll}
\hline$n$ & $\theta=0.5$ & $\theta=1$ & $\theta=1.5$ & $\theta=2$ & $\theta=2.5$ & $\theta=3$ & $\theta=3.5$ & $\theta=4$ & $\theta=4.5$ \\
\hline 2 & 1.3041 & 1.3126 & 1.2850 & 1.2667 & 1.2565 & 1.2503 & 1.2454 & 1.2363 & 1.2296 \\
3 & 1.1105 & 1.1138 & 1.1026 & 1.0948 & 1.0892 & 1.0846 & 1.0840 & 1.0826 & 1.0813 \\
4 & 1.0578 & 1.0594 & 1.0538 & 1.0490 & 1.0455 & 1.0440 & 1.0398 & 1.0408 & 1.0643 \\
5 & 1.0357 & 1.0367 & 1.0328 & 1.0301 & 1.0278 & 1.0259 & 1.0259 & 1.0232 & 1.0048 \\
\hline
\end{tabular}

In Table 8, we presented the REC of $\hat{Y}_{B L I P}$ relative to $\hat{Y}_{B L U P}$ for different choices of $n$ and $\theta$. From Table 8, we observe that the BLIP works better than BLUP in terms of MSPE.

Suppose we are now interested in PIs for $Y_{U(n+1)}$. The PIs can be constructed using the pivotal quantities [24]

$$
T_{1}=\frac{Y_{U(n+1)}-Y_{U(n)}}{\hat{\sigma}_{B L U}}
$$

and

$$
T_{2}=\frac{Y_{U(n+1)}-Y_{U(n)}}{\hat{\sigma}_{B L I}}
$$

Constructing such PIs requires the percentage points of $T_{1}$ and $T_{2}$. In Table 9, we presented the simulated percentage points of $T_{1}$ and $T_{2}$ using Monte Carlo method based on 10000 runs and different choices of $n$ and $\theta$. Using the pivotal quantity $T_{1}$, a $100(1-\alpha) \%$ PI for $Y=Y_{U(n+1)}$ is given by

$$
P\left(Y_{U(n)}+\hat{\sigma}_{B L U} T_{1}(1-\alpha / 2) \leq Y \leq Y_{U(n)}+\hat{\sigma}_{B L U} T_{1}(\alpha / 2)\right)=1-\alpha .
$$

Similarly, using the pivotal quantity $T_{2}$, a $100(1-\alpha) \%$ PI for $Y$ is given by

$$
P\left(Y_{U(n)}+\hat{\sigma}_{B L I} T_{2}(1-\alpha / 2) \leq Y \leq Y_{U(n)}+\hat{\sigma}_{B L I} T_{2}(\alpha / 2)\right)=1-\alpha .
$$

\begin{tabular}{|c|c|c|c|c|c|c|c|c|c|}
\hline \multirow[b]{2}{*}{$\theta$} & \multirow[b]{2}{*}{$n$} & \multicolumn{4}{|c|}{$T_{1}$} & \multicolumn{4}{|c|}{$T_{2}$} \\
\hline & & $2.5 \%$ & $5 \%$ & $95 \%$ & $97.5 \%$ & $2.5 \%$ & $5 \%$ & $95 \%$ & $97.5 \%$ \\
\hline \multirow[t]{4}{*}{0.5} & 2 & 0.0712 & 0.1413 & 39.647 & 76.692 & 0.1322 & 0.2622 & 73.555 & 142.290 \\
\hline & 3 & 0.0601 & 0.1207 & 14.392 & 20.527 & 0.0768 & 0.1837 & 22.327 & 32.855 \\
\hline & 4 & 0.0614 & 0.1257 & 10.878 & 15.6182 & 0.0782 & 0.1568 & 14.165 & 20.079 \\
\hline & 5 & 0.0516 & 0.1104 & 9.6559 & 12.550 & 0.0705 & 0.1456 & 11.467 & 15.348 \\
\hline \multirow[t]{4}{*}{1} & 2 & 0.0339 & 0.0681 & 20.278 & 42.555 & 0.0609 & 0.1228 & 40.294 & 88.631 \\
\hline & 3 & 0.0334 & 0.0660 & 7.1150 & 10.249 & 0.0399 & 0.0851 & 10.712 & 16.057 \\
\hline & 4 & 0.0312 & 0.0626 & 5.4481 & 7.5059 & 0.0384 & 0.0780 & 7.2854 & 10.282 \\
\hline & 5 & 0.0280 & 0.0555 & 4.7133 & 6.2944 & 0.0356 & 0.0740 & 5.7481 & 7.7365 \\
\hline \multirow[t]{3}{*}{1.5} & 2 & 0.0225 & 0.0451 & 13.594 & 26.469 & 0.0435 & 0.0874 & 25.909 & 54.927 \\
\hline & 3 & 0.0206 & 0.0452 & 4.9984 & 7.7554 & 0.0332 & 0.0633 & 7.2247 & 11.462 \\
\hline & 4 & 0.0205 & 0.0412 & 3.6181 & 5.0762 & 0.0281 & 0.0555 & 4.8975 & 6.6060 \\
\hline
\end{tabular}

Table 9 Simulated percentage points of $T_{1}$ and $T_{2}$. 
Table 9 Simulated percentage points of $T_{1}$ and $T_{2}$. (Continued)

\begin{tabular}{|c|c|c|c|c|c|c|c|c|c|}
\hline \multirow[b]{2}{*}{$\theta$} & \multirow[b]{2}{*}{$n$} & \multicolumn{4}{|c|}{$T_{1}$} & \multicolumn{4}{|c|}{$T_{2}$} \\
\hline & & $2.5 \%$ & $5 \%$ & $95 \%$ & $97.5 \%$ & $2.5 \%$ & $5 \%$ & $95 \%$ & $97.5 \%$ \\
\hline & 5 & 0.0190 & 0.0389 & 3.1497 & 4.2039 & 0.0236 & 0.0481 & 3.9040 & 5.2271 \\
\hline \multirow[t]{4}{*}{2} & 2 & 0.0154 & 0.0328 & 10.581 & 21.302 & 0.0264 & 0.0594 & 21.275 & 43.214 \\
\hline & 3 & 0.0145 & 0.0310 & 3.8087 & 3.8087 & 0.0229 & 0.0458 & 5.4379 & 8.4731 \\
\hline & 4 & 0.0147 & 0.0304 & 2.6935 & 3.6855 & 0.0208 & 0.0415 & 3.5587 & 5.0637 \\
\hline & 5 & 0.0144 & 0.0297 & 2.3256 & 3.1242 & 0.0202 & 0.0376 & 2.9245 & 3.9670 \\
\hline \multirow[t]{4}{*}{2.5} & 2 & 0.0129 & 0.0258 & 8.0905 & 16.145 & 0.0264 & 0.0515 & 16.379 & 34.770 \\
\hline & 3 & 0.0110 & 0.0235 & 2.9614 & 4.6853 & 0.0183 & 0.0359 & 4.3968 & 7.0489 \\
\hline & 4 & 0.0116 & 0.0224 & 2.2877 & 3.0986 & 0.0155 & 0.0311 & 2.9313 & 4.0433 \\
\hline & 5 & 0.0115 & 0.0223 & 1.9293 & 2.5587 & 0.0141 & 0.0278 & 2.3385 & 3.2454 \\
\hline \multirow[t]{4}{*}{3} & 2 & 0.0107 & 0.0208 & 6.7242 & 14.437 & 0.0188 & 0.0393 & 13.919 & 30.501 \\
\hline & 3 & 0.0105 & 0.0208 & 2.3687 & 3.5040 & 0.0129 & 0.0280 & 3.8110 & 5.7746 \\
\hline & 4 & 0.0096 & 0.0193 & 1.9203 & 2.7296 & 0.0112 & 0.0245 & 2.4994 & 3.5728 \\
\hline & 5 & 0.0091 & 0.0184 & 1.6067 & 2.1887 & 0.0111 & 0.0223 & 1.9888 & 2.7872 \\
\hline \multirow[t]{4}{*}{3.5} & 2 & 0.0089 & 0.0184 & 5.7860 & 12.1082 & 0.0185 & 0.0359 & 11.736 & 24.746 \\
\hline & 3 & 0.0082 & 0.0179 & 2.1454 & 3.3560 & 0.0132 & 0.0271 & 3.2235 & 5.1909 \\
\hline & 4 & 0.0081 & 0.0175 & 1.5263 & 2.1420 & 0.0114 & 0.0226 & 2.0896 & 2.8773 \\
\hline & 5 & 0.0079 & 0.0164 & 1.3732 & 1.8560 & 0.0108 & 0.0207 & 1.6497 & 2.3159 \\
\hline \multirow[t]{4}{*}{4} & 2 & 0.0078 & 0.0154 & 5.4120 & 11.3077 & 0.0137 & 0.0288 & 10.4154 & 23.0863 \\
\hline & 3 & 0.0074 & 0.0151 & 1.9758 & 2.7955 & 0.0102 & 0.0217 & 2.8633 & 4.4407 \\
\hline & 4 & 0.0071 & 0.0140 & 1.3406 & 1.8174 & 0.0090 & 0.0190 & 1.8185 & 2.5268 \\
\hline & 5 & 0.0070 & 0.0144 & 1.1706 & 1.5569 & 0.0079 & 0.0171 & 1.4869 & 2.0726 \\
\hline \multirow[t]{4}{*}{4.5} & 2 & 0.0071 & 0.0139 & 4.6528 & 9.6756 & 0.0141 & 0.0274 & 9.1362 & 18.999 \\
\hline & 3 & 0.0058 & 0.0122 & 1.6271 & 2.4055 & 0.0093 & 0.0191 & 2.4354 & 3.6441 \\
\hline & 4 & 0.0069 & 0.0137 & 1.2583 & 1.7334 & 0.0083 & 0.0168 & 1.6336 & 2.3774 \\
\hline & 5 & 0.0067 & 0.0128 & 1.0487 & 1.3822 & 0.0077 & 0.0157 & 1.3140 & 1.7646 \\
\hline
\end{tabular}

\section{ILLUSTRATIVE EXAMPLES}

In this section, we present two numerical examples for illustrative purposes.

\subsection{Example 1 (Real Data)}

Here, we consider the total annual rainfall (in inches) during March recorded at Los Angeles Civic Center from 1972 to 2006 (see the website of Los Angeles Almanac: www.laalmanac.com/weather/we08aa.htm). From these data, we observe the upper record values as follows:

$2.70,3.78,4.83,8.02,8.37$.

A simple plot of these five upper record values against the expected values in Table 1 for $\theta=1$ indicates a very strong correlation (correlation coefficient as high as 0.972 ). Hence, the assumption that these record values come from a Lindley distribution with $\theta=1$ is quite reasonable. 
Based on these data and Tables 3 and 4, we find the BLUEs of $\mu$ and $\sigma$.

$$
\begin{aligned}
\hat{\mu}_{B L U} & =a_{1} Y_{U(1)}+a_{2} Y_{U(2)}+a_{3} Y_{U(3)}+a_{4} Y_{U(4)}+a_{5} Y_{U(5)} \\
& =(1.2710 \times 2.70)+(0.0347 \times 3.78)+(0.0171 \times 4.83)+(0.0090 \times 8.02)-(0.3320 \times 8.37) \\
& =0.9388 \\
\hat{\sigma}_{B L U} & =b_{1} Y_{U(1)}+b_{2} Y_{U(2)}+b_{3} Y_{U(3)}+b_{4} Y_{U(4)}+b_{5} Y_{U(5)} \\
& =(-0.2013 \times 2.70)+(-0.0039 \times 3.78)+(-0.0007 \times 4.83)+(0.0003 \times 8.02)+(0.2057 \times 8.37) \\
& =1.1625
\end{aligned}
$$

The corresponding variances and covariances of $\hat{\mu}_{B L U}$ and $\hat{\sigma}_{B L U}$ (see Table 5) are computed to be:

$$
\operatorname{Var}\left(\hat{\mu}_{B L U}\right)=2.3899 \sigma^{2}, \quad \operatorname{Var}\left(\hat{\sigma}_{B L U}\right)=0.2157 \sigma^{2}, \quad \operatorname{Cov}\left(\hat{\mu}_{B L U}, \hat{\sigma}_{B L U}\right)=-0.3759 \sigma^{2} .
$$

The BLIEs of the location and scale parameters are given by $\hat{\mu}_{B L I}=1.2982$ and $\hat{\sigma}_{B L I}=0.9562$. The variances of $\hat{\mu}_{B L I}$ and $\hat{\sigma}_{B L I}$ are

$$
\operatorname{Var}\left(\hat{\mu}_{B L I}\right)=2.2943 \sigma^{2}, \operatorname{Var}\left(\hat{\sigma}_{B L I}\right)=0.1459 \sigma^{2}
$$

From Eqs. (6) and (8) and use of Tables 6 and 7, the 95\% CIs for $\mu$ based on $R_{1}$ and $R_{3}$ are $(-6.3534,2.3926)$ and $(-7.5330,2.6300)$, respectively. Also from Eqs. (7) and (9), the 95\% CIs for $\sigma$ based on $R_{2}$ and $R_{4}$ are $(0.5633,3.8827)$ and $(0.6786,4.6015)$, respectively.

Suppose that we want to find the BLUP of the next record $Y_{U(6)}(n+1=6)$ based on the first $n=5$ observed records. From Table 1 we have $\alpha_{n+1}=\alpha_{6}=7.520$ when $\theta=1$. From Table 2 for $\theta=1$, we have

$$
\begin{aligned}
\omega^{\prime} & =\left(\operatorname{Cov}\left(X_{U(1)}, X_{U(6)}\right), \cdots, \operatorname{Cov}\left(X_{U(5)}, X_{U(6)}\right)\right) \\
& =(1.4709,2.7500,3.9603,5.1387,6.2996),
\end{aligned}
$$

the vector of observed records is $\mathbf{Y}^{\prime}=(2.70,3.78,4.83,8.02,8.37)$ and the vector of standard means is (from Table 1)

$$
\alpha^{\prime}=(1.500,2.819,4.052,5.236,6.389) \text {. }
$$

$\beta_{5 \times 5}$ is the variance-covariance matrix of the first five standard records which can obtained from Table 2 . The BLUP of $Y_{U(6)}$ is $\hat{Y}_{B L U P}=9.6840$. Also the BLIP of $Y_{U(6)}$ is $\hat{Y}_{B L I P}=9.4368$. In addition, using Eqs. (10) and (11) and use of Table 9, the 95\% PIs for the next upper record $Y_{U(6)}$ based on the pivotal quantity $T_{1}$ and $T_{2}$ are computed as

$$
\left(L_{1}\left(Y_{U(6)}\right), U_{1}\left(Y_{U(6)}\right)\right)=(8.4025,15.687)
$$

and

$$
\left(L_{2}\left(Y_{U(6)}\right), U_{2}\left(Y_{U(6)}\right)\right)=(8.4040,15.768)
$$

respectively.

\subsection{Example 2 (Simulated Data)}

For given values of $\theta=1.5, \mu=0$ and $\sigma=1$, we generated $n=5$ upper record values from the Lindley distribution as follows:

$1.0773,3.1755,3.4962,4.5126,4.6015$.

The BLUEs of $\mu$ and $\sigma$ are computed to be $\hat{\mu}_{B L U}=0.0934$ and $\hat{\sigma}_{B L U}=1.0925$. The corresponding variances and covariances of $\hat{\mu}_{B L U}$ and $\hat{\sigma}_{B L U}$ are computed to be:

$$
\operatorname{Var}\left(\hat{\mu}_{B L U}\right)=0.9718, \quad \operatorname{Var}\left(\hat{\sigma}_{B L U}\right)=0.2201, \quad \operatorname{Cov}\left(\hat{\mu}_{B L U}, \hat{\sigma}_{B L U}\right)=0.2332
$$

The BLIEs and the corresponding variances are

$$
\begin{gathered}
\hat{\mu}_{B L I}=0.3022, \hat{\sigma}_{B L I}=0.8954 \\
\operatorname{Var}\left(\hat{\mu}_{B L I}\right)=0.9352, \operatorname{Var}\left(\hat{\sigma}_{B L I}\right)=0.1478 .
\end{gathered}
$$


The $95 \%$ CIs for $\mu$ based on $R_{1}$ and $R_{3}$ are $(-4.8243,1.0841)$ and $(-4.8614,1.847)$, respectively. Also, the $95 \%$ CIs for $\sigma$ based on $R_{2}$ and $R_{4}$ are $(0.5179,3.6608)$ and $(0.6272,4.4363)$, respectively.

Let us now consider the BLUP and BLIP of the next record, $Y_{U(6)}$. The BLUP and BLIP are $\hat{Y}_{B L U P}=5.4264$ and $\hat{Y}_{B L I P}=5.2712$, respectively. Moreover, the $95 \%$ PIs for the next upper record $Y_{U(6)}$ based on the pivotal quantities $T_{1}$ and $T_{2}$ are computed as

$$
\left(L_{1}\left(Y_{U(6)}\right), U_{1}\left(Y_{U(6)}\right)\right)=(4.6223,9.1944)
$$

and

$$
\left(L_{2}\left(Y_{U(6)}\right), U_{2}\left(Y_{U(6)}\right)\right)=(4.6226,9.2820)
$$

respectively.

\section{SIMULATION}

In this section, we carry out an intensive Monte Carlo simulation to compare different CIs presented in Section 3. In this simulation, we have randomly generated 10000 upper record sample $Y_{U(1)}, Y_{U(2)} \cdots, Y_{U(n)}$ from the standard Lindley distribution with different choices of $n$ and $\theta$. We then computed the $95 \%$ CIs for the location parameter $\mu$ based on the pivotal quantities $R_{1}$ and $R_{3}$. We also computed the $95 \%$ CIs for the scale parameter $\mu$ based on the pivotal quantities $R_{2}$ and $R_{4}$. Table 10 presents the average confidence lengths and the corresponding coverage probabilities over 10000 replications.

From Table 10, it is observed that the average lengths of CIs for $\mu$ and $\sigma$ using BLUEs (the pivotal quantities $R_{1}$ and $R_{2}$ ) are smaller than the corresponding average lengths obtained using BLIEs (the pivotal quantities $R_{3}$ and $R_{4}$ ). Also, the coverage probabilities of all CIs are quite close to the nominal level 95\%. Also, all the CIs lengths decrease as $n$ increases.

We also computed the 95\% PIs for $Y=Y_{U(n+1)}$ based on the upper record sample $Y_{U(1)}, Y_{U(2)} \cdots, Y_{U(n)}$, by using the pivotal quantities $T_{1}$ and $T_{2}$. For various choices of $n$ and $\theta$, Table 11 presents the means and coverage probabilities of the lengths of the PIs. From Table 11 , we note that the average lengths of the PIs using BLUP (the pivotal quantity $T_{1}$ ) are smaller than the corresponding lengths obtained using BLIP (the pivotal quantity $T_{2}$ ). Also, the lengths of PIs decrease as $n$ increases.

\begin{tabular}{|c|c|c|c|c|c|c|c|c|c|}
\hline \multirow[b]{3}{*}{$\theta$} & \multirow[b]{3}{*}{$n$} & \multicolumn{4}{|c|}{$\mu$} & \multicolumn{4}{|c|}{$\sigma$} \\
\hline & & \multicolumn{2}{|c|}{$R_{1}$} & \multicolumn{2}{|c|}{$R_{3}$} & \multicolumn{2}{|c|}{$R_{2}$} & \multicolumn{2}{|r|}{$R_{4}$} \\
\hline & & $\mathrm{AL}$ & $\mathrm{CP}$ & $\mathrm{AL}$ & $\mathrm{CP}$ & $\mathrm{AL}$ & $\mathrm{CP}$ & Average Length & Coverage Probability \\
\hline \multirow[t]{4}{*}{0.5} & 2 & 116.968 & 0.9541 & 133.501 & 0.9490 & 32.1525 & 0.9533 & 63.0546 & 0.9454 \\
\hline & 3 & 31.4461 & 0.9515 & 33.4645 & 0.9499 & 6.3723 & 0.9527 & 9.1189 & 0.9490 \\
\hline & 4 & 22.1436 & 0.9526 & 23.0607 & 0.9500 & 3.8845 & 0.9541 & 4.7927 & 0.9507 \\
\hline & 5 & 17.9984 & 0.9533 & 18.1236 & 0.9501 & 2.7875 & 0.9566 & 3.3262 & 0.9468 \\
\hline \multirow[t]{4}{*}{1} & 2 & 60.5284 & 0.9596 & 61.3064 & 0.9498 & 39.1433 & 0.9532 & 74.4361 & 0.9436 \\
\hline & 3 & 14.7719 & 0.9469 & 15.4661 & 0.9499 & 6.8716 & 0.9515 & 10.1473 & 0.9521 \\
\hline & 4 & 10.2411 & 0.9481 & 10.4097 & 0.9499 & 3.9601 & 0.9481 & 4.9239 & 0.9544 \\
\hline & 5 & 8.7077 & 0.9509 & 8.8175 & 0.9510 & 2.8429 & 0.9485 & 3.4934 & 0.9450 \\
\hline \multirow[t]{4}{*}{1.5} & 2 & 33.2921 & 0.9498 & 34.8740 & 0.9514 & 34.3637 & 0.9477 & 66.8064 & 0.9550 \\
\hline & 3 & 9.0784 & 0.9544 & 9.4398 & 0.9481 & 6.7328 & 0.9488 & 9.9251 & 0.9479 \\
\hline & 4 & 6.5841 & 0.9496 & 6.8003 & 0.9492 & 4.0801 & 0.9505 & 5.0701 & 0.9515 \\
\hline & 5 & 5.4468 & 0.9511 & 5.4691 & 0.9500 & 2.8973 & 0.9502 & 3.5035 & 0.9457 \\
\hline \multirow[t]{4}{*}{2} & 2 & 27.1318 & 0.9499 & 27.8236 & 0.9511 & 40.2612 & 0.9501 & 79.1642 & 0.9483 \\
\hline & 3 & 6.8626 & 0.9499 & 7.0130 & 0.9512 & 7.1925 & 0.9503 & 10.7277 & 0.9519 \\
\hline & 4 & 4.6924 & 0.9498 & 4.7060 & 0.9472 & 3.9994 & 0.9501 & 5.2650 & 0.9438 \\
\hline & 5 & 3.8978 & 0.9500 & 3.9028 & 0.9503 & 2.8952 & 0.9500 & 3.6018 & 0.9537 \\
\hline
\end{tabular}

Table 10 Average length (ALs) and coverage probabilities (CPs) of 95\% CIs based on $R_{1}, R_{2}, R_{3}$ and $R_{4}$. 
Table 10 Average length (ALs) and coverage probabilities (CPs) of $95 \%$ CIs based on $R_{1}, R_{2}, R_{3}$ and $R_{4}$. (Continued)

\begin{tabular}{|c|c|c|c|c|c|c|c|c|c|}
\hline \multirow[b]{3}{*}{$\theta$} & \multirow[b]{3}{*}{$n$} & \multicolumn{4}{|c|}{$\mu$} & \multicolumn{4}{|c|}{$\sigma$} \\
\hline & & \multicolumn{2}{|c|}{$R_{1}$} & \multicolumn{2}{|c|}{$R_{3}$} & \multicolumn{2}{|c|}{$R_{2}$} & \multicolumn{2}{|r|}{$R_{4}$} \\
\hline & & $\mathrm{AL}$ & $\mathrm{CP}$ & $\mathrm{AL}$ & $\mathrm{CP}$ & $\mathrm{AL}$ & $\mathrm{CP}$ & Average Length & Coverage Probability \\
\hline \multirow[t]{4}{*}{2.5} & 2 & 18.2254 & 0.9498 & 19.3539 & 0.9482 & 35.4389 & 0.9501 & 72.0573 & 0.9504 \\
\hline & 3 & 5.0056 & 0.9500 & 5.1745 & 0.9492 & 7.0564 & 0.9500 & 11.4473 & 0.9518 \\
\hline & 4 & 3.6115 & 0.9499 & 3.8093 & 0.9540 & 4.2128 & 0.9502 & 5.4784 & 0.9550 \\
\hline & 5 & 3.0177 & 0.9481 & 3.0396 & 0.9500 & 2.9284 & 0.9498 & 3.6385 & 0.9458 \\
\hline \multirow[t]{4}{*}{3} & 2 & 15.8027 & 0.9499 & 16.1069 & 0.9489 & 40.6387 & 0.9501 & 74.1516 & 0.9499 \\
\hline & 3 & 4.1521 & 0.9484 & 4.2726 & 0.9488 & 7.2285 & 0.9503 & 11.1147 & 0.9467 \\
\hline & 4 & 2.9453 & 0.9510 & 2.9559 & 0.9476 & 4.3355 & 0.9533 & 5.4733 & 0.9553 \\
\hline & 5 & 2.4669 & 0.9458 & 2.4707 & 0.9482 & 2.9882 & 0.9454 & 3.6971 & 0.9566 \\
\hline \multirow[t]{4}{*}{3.5} & 2 & 12.3725 & 0.9499 & 14.1363 & 0.9500 & 35.9585 & 0.9501 & 72.8842 & 0.9510 \\
\hline & 3 & 3.3948 & 0.9499 & 3.6863 & 0.9470 & 7.2159 & 0.9500 & 11.6175 & 0.9536 \\
\hline & 4 & 2.4362 & 0.9502 & 2.5758 & 0.9534 & 4.2576 & 0.9500 & 5.3757 & 0.9537 \\
\hline & 5 & 2.0773 & 0.9499 & 2.0885 & 0.9514 & 3.0120 & 0.9501 & 3.6965 & 0.9555 \\
\hline \multirow[t]{4}{*}{4} & 2 & 12.1945 & 0.9500 & 12.5072 & 0.9506 & 41.1703 & 0.9496 & 72.9495 & 0.9451 \\
\hline & 3 & 3.0108 & 0.9498 & 3.2115 & 0.9479 & 7.3866 & 0.9501 & 11.6581 & 0.9485 \\
\hline & 4 & 2.1256 & 0.9498 & 2.1618 & 0.9509 & 4.1769 & 0.9501 & 5.5760 & 0.9502 \\
\hline & 5 & 1.7783 & 0.9499 & 1.7858 & 0.9502 & 3.0125 & 0.9501 & 3.7677 & 0.9483 \\
\hline \multirow[t]{4}{*}{4.5} & 2 & 9.2964 & 0.9499 & 10.6092 & 0.9504 & 36.1874 & 0.9500 & 74.7037 & 0.9490 \\
\hline & 3 & 2.5535 & 0.9499 & 2.7794 & 0.9493 & 7.3018 & 0.9500 & 11.6565 & 0.9506 \\
\hline & 4 & 1.8548 & 0.9500 & 1.8588 & 0.9492 & 4.2612 & 0.9501 & 5.4964 & 0.9504 \\
\hline & 5 & 1.5655 & 0.9500 & 1.5823 & 0.9509 & 3.0399 & 0.9502 & 3.6726 & 0.9527 \\
\hline
\end{tabular}

Table 11 Average length (ALs) and coverage probabilities (CPs) of 95\% CIs based on $T_{1}$ and $T_{2}$.

\begin{tabular}{|c|c|c|c|c|c|}
\hline \multirow[b]{2}{*}{$\theta$} & \multirow[b]{2}{*}{$n$} & \multicolumn{2}{|c|}{$T_{1}$} & \multicolumn{2}{|c|}{$T_{2}$} \\
\hline & & $\mathrm{AL}$ & $\mathrm{CP}$ & $\mathrm{AL}$ & $\mathrm{CP}$ \\
\hline \multirow[t]{4}{*}{0.5} & 2 & 77.144 & 0.9543 & 77.145 & 0.9522 \\
\hline & 3 & 20.512 & 0.9419 & 23.092 & 0.9449 \\
\hline & 4 & 15.647 & 0.9431 & 15.699 & 0.9435 \\
\hline & 5 & 12.397 & 0.9467 & 12.513 & 0.9463 \\
\hline \multirow[t]{4}{*}{1} & 2 & 42.748 & 0.9471 & 47.2928 & 0.9455 \\
\hline & 3 & 10.248 & 0.9532 & 11.199 & 0.9534 \\
\hline & 4 & 7.5315 & 0.9449 & 7.9066 & 0.9449 \\
\hline & 5 & 6.2920 & 0.9485 & 6.3604 & 0.9467 \\
\hline \multirow[t]{4}{*}{1.5} & 2 & 26.548 & 0.9574 & 28.921 & 0.9466 \\
\hline & 3 & 7.7232 & 0.9535 & 7.8957 & 0.9440 \\
\hline & 4 & 5.0579 & 0.9579 & 5.0833 & 0.9560 \\
\hline & 5 & 4.1784 & 0.9553 & 4.2582 & 0.9491 \\
\hline \multirow[t]{4}{*}{2} & 2 & 21.192 & 0.9483 & 22.376 & 0.9442 \\
\hline & 3 & 3.7841 & 0.9437 & 5.7966 & 0.9441 \\
\hline & 4 & 3.6817 & 0.9473 & 3.8702 & 0.9423 \\
\hline & 5 & 3.096 & 0.9455 & 3.2107 & 0.9477 \\
\hline
\end{tabular}


Table 11 Average length (ALs) and coverage probabilities (CPs) of 95\% CIs based on $T_{1}$ and $T_{2}$. (Continued)

\begin{tabular}{|c|c|c|c|c|c|}
\hline \multirow[b]{2}{*}{$\theta$} & \multirow[b]{2}{*}{$n$} & \multicolumn{2}{|c|}{$T_{1}$} & \multicolumn{2}{|c|}{$T_{2}$} \\
\hline & & $\mathrm{AL}$ & $\mathrm{CP}$ & $\mathrm{AL}$ & $\mathrm{CP}$ \\
\hline \multirow[t]{4}{*}{2.5} & 2 & 16.0882 & 0.9425 & 17.9052 & 0.9466 \\
\hline & 3 & 4.7601 & 0.9433 & 4.8020 & 0.9456 \\
\hline & 4 & 3.0764 & 0.9465 & 3.0770 & 0.9467 \\
\hline & 5 & 2.5529 & 0.9480 & 2.6390 & 0.9480 \\
\hline \multirow[t]{4}{*}{3} & 2 & 14.512 & 0.9570 & 15.762 & 0.9554 \\
\hline & 3 & 3.4745 & 0.9564 & 3.6993 & 0.9578 \\
\hline & 4 & 2.7251 & 0.9522 & 2.7274 & 0.9509 \\
\hline & 5 & 2.1801 & 0.9485 & 2.2579 & 0.9482 \\
\hline \multirow[t]{4}{*}{3.5} & 2 & 12.1655 & 0.9426 & 12.7269 & 0.9464 \\
\hline & 3 & 3.3610 & 0.9458 & 3.5346 & 0.9484 \\
\hline & 4 & 2.1383 & 0.9577 & 2.1902 & 0.9546 \\
\hline & 5 & 1.8356 & 0.9521 & 1.8580 & 0.9522 \\
\hline \multirow[t]{4}{*}{4} & 2 & 11.3436 & 0.9532 & 11.8191 & 0.9535 \\
\hline & 3 & 2.7905 & 0.9555 & 3.0067 & 0.9529 \\
\hline & 4 & 1.8135 & 0.9460 & 1.9197 & 0.9450 \\
\hline & 5 & 1.5562 & 0.9446 & 1.6797 & 0.9462 \\
\hline \multirow[t]{4}{*}{4.5} & 2 & 9.6661 & 0.9527 & 9.6663 & 0.9557 \\
\hline & 3 & 2.4182 & 0.9464 & 2.4784 & 0.9458 \\
\hline & 4 & 1.7283 & 0.9457 & 1.8020 & 0.9463 \\
\hline & 5 & 1.3776 & 0.9444 & 1.4251 & 0.9417 \\
\hline
\end{tabular}

\section{COMPETING INTERESTS}

The authors declare that they have no conflict of interests.

\section{ACKNOWLEDGMENTS}

The authors are thankful to the Editor and the reviewers for recommending the paper for publication.

\section{REFERENCES}

1. R.L. Houchens. Record Value Theory and Inference, Ph.D. thesis. University of California, Riverside, 1984.

2. K.N. Chandler. J. Roy. Stat. Soc. Ser. B. 14(1952), 220-228.

3. M. Ahsanullah, Record Statistics, Nova Science Publishers, Commack, New York, 1995.

4. B.C. Arnold, N. Balakrishnan, H.N. Nagaraja. Records, John Wiley \& Sons, New York, 1998.

5. V.B. Nevzorov. Records: Mathematical Theory, 194. American Mathematical Society Providence, Rhode Island, 2001.

6. D.V. Lindley, J. Roy. Stat. Soc. Ser. B. 20(1958), 102-107.

7. M.E. Ghitany, B. Atieh, S. Nadarajah. Math. Comput. Simul. 78(2008), 493-506.

8. H. Zakerzadeh, A. Dolati, J. Math. Ext. 3(2009), 13-25.

9. M.E. Ghitany, F. Alqallaf, D.K. Al-Mutairi, H.A. Husain, Math. Comput. Simul. 81(2011), 1190-1201.

10. H.S. Bakouch, B.M. Al-Zahrani, A.A. Al-Shomrani, V.A.A. Marchi, F. Louzada. J. Korean Stat. Soc. 41(2012), 75-85.

11. M.E. Ghitany, D.K. Al-Mutairi, N. Balakrishnan, L.J. Al-Enezi,Comput. Stat. Data Anal. 64(2013), 20-33.

12. A. Asgharzadeh, H.S. Bakouch, S. Nadarajah, F. Sharafi. Braz. J. Probab. Stat. 30(2016), 1-27.

13. A. Asgharzadeh, S. Nadarajah, F. Sharafi. REVSTAT 16(2018), 87-113.

14. A. Asgharzadeh, S. Nadarajah, F. Sharafi, Commun. Stat. Theory Methods. 46(10) (2017), 5001-5021.

15. H. Krishna, K. Kumar, Math. Comput. Simulat. 82(2011), 281-294.

16. S. Ali, M. Aslam, S.M.A. Kazmi, Appl. Math. Model. 37(2013), 6068-6078.

17. P.K. Gupta, B. Singh,Int. J. Syst. Assur. Eng. Manag. 4(2013), 378-385.

18. D.K. Al-Mutairi, M.E. Ghitany, D. Kundu,Commun. Stat. Theory Methods. 42(2013), 1443-1463.

19. A. Asgharzadeh, H. K. T. Ng, R. Valiollahi, M. Azizpour, J. Stat. Theory Appl. 16(2017), 178-197. 
20. A.Asgharzadeh, A. Fallah, M.Z. Raqab, R. Valiollahi, Stat. Papers. 59(2018), 759-779.

21. M. Ahsanullah, Ann. Inst. Statist. Math. 32(1980), 363-368.

22. I.R. Dunsmore, Ann. Inst. Stat. Math. 35(1983), 267-277.

23. N. Balakrishnan, M. Ahsanullah, P.S. Chan, J. Appl. Stat. Sci. 2 (1995), 233-248.

24. N. Balakrishnan, P.S. Chan, Stat. Probabil. Lett. 39(1998), 73-80.

25. M.Z. Raqab, J. Stat. Plan. Inference. 104(2002), 339-350.

26. K.S. Sultan, G.R. AL-Dayian, H.H. Mohammad,Comput. Stat. Data Anal. 52(2008), 1430-1440.

27. S.M.T.K. MirMostafaee, A. Asgharzadeh, A. Fallah, Metron. 74(2016), 37-59.

28. I.S. Gradshteyn, I.M. Ryzhik. Table of Integrals, Series, and Products. sixth ed. Academic Press San Diego 2000.

29. N. Balakrishnan, A.C. Cohen, Order Statistics and Inference: Estimation Methods, Academic Press, San Diego, 1991.

30. N.R. Mann, Ann. Math. Stat. 40(1969), 2149-2155.

31. M. Burkschat, Commun. Stat. Theory Methods. 39(2010), 311-326. 


\section{APPENDIX A. DERIVATION OF EQ. (2) WHEN $n=m+1$.}

$$
\begin{aligned}
& \mu_{m, m+1}^{(r, s)}=\frac{\theta^{4}}{\Gamma(m)(\theta+1)} \int_{0}^{\infty} x^{r}\left\{-\log \left(\frac{\theta+1+\theta x}{\theta+1}\right)+\theta x\right\}^{m-1} \frac{1+x}{\theta+1+\theta x} \\
& \times \int_{x}^{\infty} y^{s}(1+y) e^{-\theta y} \mathrm{~d} y \mathrm{~d} x \\
& =\frac{\theta^{3}}{\Gamma(m)(\theta+1)} \int_{0}^{\infty} x^{r}\left\{-\log \left(\frac{\theta+1+\theta x}{\theta+1}\right)+\theta x\right\}^{m-1} \frac{1+x}{\theta+1+\theta x} \\
& \times\left[\frac{x^{s+1}}{s+1}+\sum_{j=0}^{s} \frac{\Gamma(s)[1+s / \theta]}{\theta^{s-j} j !} x^{j}\right] e^{-\theta x} \mathrm{~d} x \\
& =\frac{(\theta+1)^{r-1} e^{\theta+1}}{\Gamma(m) \theta^{r-1}} \int_{1}^{\infty}(u-1)^{r-1}\{-\log u+(\theta+1)(u-1)\}^{m-1}[\theta+(\theta+1)(u-1)] \\
& \times\left[\frac{[(\theta+1)(u-1) / \theta]^{s+1}}{s+1}+\sum_{j=0}^{s} \frac{\Gamma(s)[1+s / \theta]}{\theta^{s} j !}[(\theta+1)(u-1)]^{j}\right] e^{-(\theta+1) u} d u \\
& =\frac{(\theta+1)^{r-1} e^{\theta+1}}{\Gamma(m) \theta^{r-1}} \sum_{i=0}^{m-1}\left(\begin{array}{l}
m-1 \\
i
\end{array}\right)(-1)^{i}(\theta+1)^{m-i-1} \\
& \times \int_{1}^{\infty}(u-1)^{r+m-2-i}\{-\log u\}^{i}[\theta+(\theta+1)(u-1)] \\
& \times\left(\frac{[(\theta+1)(u-1) / \theta]^{s+1}}{s+1}+\sum_{j=0}^{s} \frac{\Gamma(s)[1+s / \theta]}{\theta^{s} j !}[(\theta+1)(u-1)]^{j}\right) e^{-(\theta+1) u} d u \\
& =\frac{(\theta+1)^{r-1} e^{\theta+1}}{\Gamma(m) \theta^{s+r-1}} \sum_{i=0}^{m-1}\left(\begin{array}{l}
m-1 \\
i
\end{array}\right)(-1)^{i}(\theta+1)^{m-i} \\
& \times\left\{\frac{(\theta+1)^{s}}{s+1} \sum_{q=1}^{r+m-i+s}\left(\begin{array}{l}
r+m-1-i+s \\
q-1
\end{array}\right)(-1)^{r+m+s} h(q, i, \theta)\right. \\
& +\frac{(\theta+1)^{s+1}}{(s+1) \theta} \sum_{q=1}^{r+m-i+s+1}\left(\begin{array}{l}
r+m-i+s \\
q-1
\end{array}\right)(-1)^{r+m+s+1} h(q, i, \theta) \\
& +\sum_{j=0}^{s}(\theta+1)^{j-1} \frac{\Gamma(s)[\theta+s]}{j !} \\
& \times \sum_{q=1}^{r+m-1+j-i}\left(\begin{array}{l}
r+m-2+j-i \\
q-1
\end{array}\right)(-1)^{r+m-1+j} h(q, i, \theta) \\
& \left.+\sum_{j=0}^{s}(\theta+1)^{j} \frac{\Gamma(s)[1+s / \theta]}{j !} \sum_{q=1}^{r+m+j-i}\left(\begin{array}{l}
r+m-1+j-i \\
q-1
\end{array}\right)(-1)^{r+m+j} h(q, i, \theta)\right\},
\end{aligned}
$$

where

$$
h(q, i, \theta)=(-1)^{-i-q} \frac{\partial^{i}}{\partial q^{i}}\left\{(\theta+1)^{-q} \Gamma(q, \theta+1)\right\} .
$$

